\title{
TRAF6 expression is associated with poorer prognosis and high recurrence in urothelial bladder cancer
}

\author{
HANLI WU ${ }^{1 *}$, AIXIA HAO $^{1 *}$, HONGHONG CUI $^{1}$, WENBIN WU $^{1}$, \\ HUANRONG YANG ${ }^{1}$, BAOHONG HU ${ }^{2,3}$ and PENG $\mathrm{LI}^{3}$ \\ ${ }^{1}$ Department of Nephrology, Yidu Central Hospital of Weifang, Weifang, Shandong 262500; \\ ${ }^{2}$ East District of Shandong Provincial Hospital of Shandong Univeristy, Jinan, Shandong 250014; \\ ${ }^{3}$ Department of Medical Oncology, Yantai Yuhuangding Hospital Affiliated to Qingdao University, \\ Yantai, Shandong 264000, P.R. China
}

Received September 26, 2015; Accepted April 13, 2017

DOI: $10.3892 / \mathrm{ol} .2017 .6427$

\begin{abstract}
The aim of the present study was to investigate the clinical significance of TNF receptor-associated factor 6 (TRAF6) expression in urothelial bladder cancer. TRAF6 expression was detected by immunohistochemistry in 126 samples of patients with urothelial bladder cancer. The association between clinicopathological factors and TRAF6 expression was analyzed by $\chi^{2}$ test. The association between TRAF6 expression, overall survival rate and the recurrence-free survival rate was evaluated in univariate analysis with Kaplan-Meier test and in multivariate analysis with Cox-regression model. In the cohort tested, the rate of high TRAF6 expression was $61.9 \%$ (78/126). TRAF6 expression was demonstrated to be significantly associated with positive metastasis $(\mathrm{P}=0.001)$ with $\chi^{2}$ test. Furthermore, TRAF6 expression was demonstrated to be associated with overall survival rate $(\mathrm{P}=0.016)$ and recurrence-free survival rate $(\mathrm{P}=0.016)$. With Cox-regression model, it was indicate that TRAF6 high expression was an independent predictive factor of poor prognosis $(\mathrm{P}=0.037)$ and high recurrence $(\mathrm{P}=0.011)$. High TRAF6 expression may predict unfavorable prognosis and high recurrence in urothelial bladder cancer, indicating that TRAF6 may be a potential and promising therapeutic target in urothelial bladder cancer.
\end{abstract}

Correspondence to: Dr Baohong Hu or Dr Peng Li, Department of Medical Oncology, Yantai Yuhuangding Hospital Affiliated to Qingdao University, 20 Yuhuangding East Road, Yantai, Shandong 264000, P.R. China

E-mail:khan2017@126.com

E-mail: lipengyuhuangding@163.com

*Contributed equally

Key words: TNF receptor-associated factor 6, recurrence, prognosis, metastasis, urothelial bladder cancer

\section{Introduction}

Bladder cancer is the most frequent malignancy of urinary tract and the 11th most common cancer with an incidence of 5.3 cases per 100,000 worldwide (1). Compared with developing countries, bladder cancer poses an increased risk to health due to its higher incidence in developed countries (2). Bladder cancer is the fifth most common type of cancer in Europe with 116,000 new cases per year (3).

There are various histological types of bladder cancer, including urothelial bladder cancer, squamous carcinoma and adenocarcinoma, of which urothelial bladder cancer is the most frequent type, accounting for $>90 \%$ of cases (4). Clinically, bladder cancer can be divided into non-muscle-invasive bladder cancer (NMIBC) and muscle-invasive bladder cancer (MIBC), with different treatments and outcome (4). NMIBC accounts for $\sim 75-85 \%$ of all bladder cancers, with relatively higher overall survival rates compared with MIBC (5).

At diagnosis, 15-25\% of patients with bladder cancer have muscle-invasion. Although there have been advances in radical cystectomy and systemic therapy, $~ 50 \%$ of these patients with muscle-invasion succumb from metastatic disease $(6,7)$. Overall, $\sim 50-80 \%$ of all treated patients with transurethral resection have tumor relapse within a period of 2 years and $10-25 \%$ of cases may present with a high grade tumor (8). Discovery of novel effective prognostic or predictive biomarkers may enable the development of therapeutics. Therefore, it is urgently required to search for novel effective and promising biomarkers, which may decrease relapse rate and improve overall survival rate.

Tumor necrosis factor (TNF) receptor-associated factor 6 (TRAF6) is a member of a new family of signaling proteins with a conserved TRAF domain of $\sim 230$ amino acids in the C-terminal and zinc finger motifs in the N-terminal (9) TRAF6 may function as an important molecule in activating nuclear factor- $\kappa \mathrm{B}(\mathrm{NF}-\kappa \mathrm{B})$ by mediating interactions to NF- $\kappa \mathrm{B}(10)$. Inhibition of TRAF6 by short interfering RNA has been demonstrated in in vitro experiments to impair interleukin-1 (IL-1)-induced NF- $\mathrm{KB}$ and c-Jun/activator protein 1 activation. This leads to the inhibition of proliferation and promotion of apoptosis in myeloma cells $(11,12)$. Furthermore, TRAF6 
is an E3 ubiquitin ligase, which mediates the synthesis of Lysine-63-linked-polyubiquitin chains conjugated to proteins, including $\mathrm{I} \kappa \mathrm{B}$ kinase subunit $\gamma(\mathrm{IKBKG})$, interleukin 1 receptor associated kinase 1 (IRAK1), AKT1, AKT2, ubiquitin conjugating enzyme E2 $\mathrm{N}$ and ubiquitin conjugating enzyme E2 V1 (13). Previously, TRAF6 has been demonstrated to promote oncogenesis by inhibiting apoptosis and stimulating proliferation and invasion as an E3 ubiquitin ligase $(14,15)$. Additionally, overexpression of TRAF6 was observed in a number of types of malignancies, including glioma, pancreatic cancer and non-small cell lung cancer (16-18). However, the clinical significance and biological effects of TRAF6 remain to be elucidated in bladder cancer.

In the present study, the expression of TRAF6 was detected in 126 cases of urothelial bladder cancer and the cohort was divided according to TRAF6 expression into low and high expression groups. The associations between TRAF6 and clinicopathological factors were analyzed using $\chi^{2}$ test. Furthermore, the effects of TRAF6 expression on tumor recurrence were analyzed in univariate and multivariate analyses. In addition, we evaluated the associations between TRAF6 expression and other clinicopathological factors.

\section{Patients and methods}

Patients and sample collection. Between January, 2005 to December, 2012, a total of 329 patients were diagnosed as bladder cancer and underwent surgical resection (transurethral tumor resection or radical total bladder cystectomy) at the Yidu Central Hospital of Weifang (Weifang, China), which were considered as the primary cohort of the present study.

A total of 126 patients with MIBC and NMIBC were selected for the validation cohort if the following criteria were fulfilled: i) The histological type is urothelial bladder cancer, ii) the patient had follow-ups following surgery, and paraffin-embedded samples were available, and iii) no history of other tumors and no neoadjuvant chemotherapy. The validation cohort consisted of 97 patients with tumor stage Ta-T according to the American Joint Committee on Cancer/Union for International Cancer Control staging system (19), who underwent transurethral tumor resection and 29 patients with tumor stage T2-T4, who underwent radical total bladder cystectomy. All 126 paraffin-embedded samples were obtained from the Pathological Department at the Yidu Central Hospital of Weifang as part of routine examination, with prior informed consent of all patients and prior approval of the Ethics Committee of Yidu Central Hospital of Weifang and Shandong Cancer Hospital and Institute.

The parameters used to evaluate the clinical significance of TRAF6 expression in the present study are overall survival rate and recurrence-free survival rate. Recurrence was defined as any new bladder cancer lesion detected following surgery, and the time of recurrence was defined from time of surgery to diagnosis of new tumors.

Immunohistochemical (IHC) staining. The tissue samples were deparaffinized for $20 \mathrm{~min}$ and rehydrated in graded ethanol according to previous studies (20-22). The samples were subsequently incubated in $0.01 \mathrm{M}$ citric acid $(\mathrm{pH} 6.0)$ and heated in a microwave for $30 \mathrm{~min}$ for antigen retrieval.
Endogenous peroxidase activity was blocked by incubating the samples in $0.3 \%$ hydrogen peroxide at room temperature for 10 mins. The slides were incubated in diluted anti-TRAF6 primary antibody (1:50; cat. no. sc-8409, Santa Cruz Biotechnology, Inc., Dallas, TX, USA) at $4^{\circ} \mathrm{C}$ overnight, followed by primary antibody retrieval and washed with phosphate buffered saline (PBS). The slides were incubated in horseradish peroxidase-labeled anti-mouse secondary antibody at dilution 1:1,000 (cat. no. A0216, Beyotime Institute of Biotechnology, Haimen, China) at $37^{\circ} \mathrm{C}$ for $2 \mathrm{~h}$. Subsequently, the slides were incubated with 3,3'-diaminobenzidine for visualization, and the slides were counterstained with hematoxylin at room temperature for $5 \mathrm{~min}$.

Staining was scored by two senior pathologists, who were unaware of the clinical information of the patients. The samples were scored according to staining intensity and the percentage of positively stained cells, which has been described previously $(23,24)$. The staining intensity was scored as weak (score 1), medium (score 2) or high (score 3 ). The scores for positively stained cells were determined as follows: $25 \%$ (score 1), 25-75\% (score 2) and $75 \%$ (score 3 ). The final IHC score was calculated as the intensity score multiplied by the score for positively stained cells, which ranged from 1 to 9 (25). Samples with final scores of $\geq 4$ were defined as high TRAF6 expression and final scores of $<4$ as low TRAF6 expression.

Statistical analysis. The software SPSS 17.0 (SPSS, Inc., Chicago, IL, USA) was used to analyze all the data and generate the P-values. The associations between TRAF6 expression and clinicopathological features were evaluated by $\chi^{2}$ test. Kaplan-Meier analysis and log-rank test were performed for analysis of recurrence-free survival and overall survival curves and to evaluate statistical difference. In multivariate analysis, the Cox-regression proportional hazards model was used to identify the independent factors. In all tests, $\mathrm{P}<0.05$ was considered to indicate a statistically significant difference.

\section{Results}

Expression of TRAF6 in bladder cancer tissues. Expression of TRAF6 was initially detected by IHC. As a signal mediator of members of the TNF receptor superfamily and members of the Toll/interleukin (IL)-1 family, TRAF6 was primarily expressed in the cytoplasm of bladder cancer cells. IHC results were evaluated by calculating the total IHC score, which was the product of staining intensity and the percentage of positively stained cells as described previously in the Patients and materials section. The validation cohort was further divided according to the level of TRAF6 expression (low and high expression groups), according to the IHC score (Fig. 1). In the present cohort, the rates of low and high TRAF6 expression were $38.10 \%$ (48/126) and $61.90 \%$ (78/126), respectively.

Association between TRAF6 expression and clinicopathological factors. $\chi^{2}$ test was used to evaluate the association between TRAF6 expression and clinicopathological factors, including sex, age, tumor diameter, tumor number, tumor 
Low expression of TRAF6

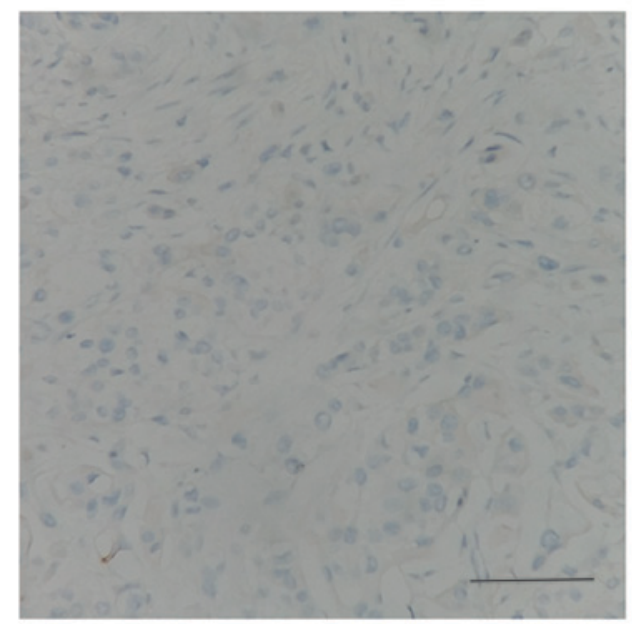

Figure 1. Representative immunohistochemical images of low and high TRAF6 expression. Scale bars, $100 \mu \mathrm{m}$.
High expression of TRAF6

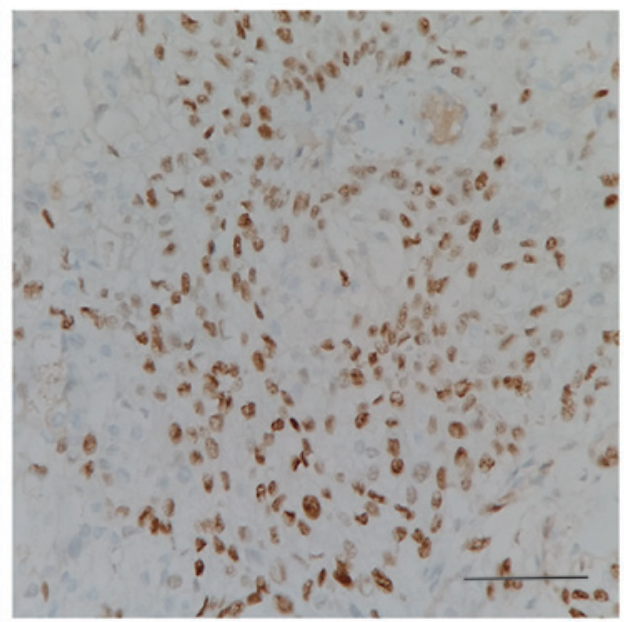

Table I. Associations between TRAF6 expression and clinicopathological factors in urothelial bladder cancer.

\begin{tabular}{|c|c|c|c|c|}
\hline \multirow[b]{2}{*}{ Parameters } & \multirow[b]{2}{*}{$\mathrm{n}$} & \multicolumn{2}{|c|}{$\begin{array}{c}\text { TRAF6 } \\
\text { expression }\end{array}$} & \multirow[b]{2}{*}{ P-value ${ }^{a}$} \\
\hline & & Low & High & \\
\hline $\operatorname{Sex}$ & & & & 0.229 \\
\hline Male & 99 & 35 & 64 & \\
\hline Female & 27 & 13 & 14 & \\
\hline Age & & & & 0.246 \\
\hline$<60$ & 43 & 13 & 30 & \\
\hline$\geq 60$ & 63 & 15 & 48 & \\
\hline Tumor diameter, $\mathrm{cm}$ & & & & 0.856 \\
\hline$\leq 3$ & 64 & 25 & 39 & \\
\hline$>3$ & 62 & 23 & 39 & \\
\hline Tumor number & & & & 0.218 \\
\hline Single & 105 & 43 & 62 & \\
\hline Multiple & 21 & 5 & 16 & \\
\hline Metastasis status & & & & 0.001 \\
\hline Negative & 116 & 48 & 68 & \\
\hline Positive & 10 & 0 & 10 & \\
\hline Tumor stage & & & & 0.828 \\
\hline Ta-T1 & 97 & 38 & 59 & \\
\hline T2-T4 & 29 & 10 & 19 & \\
\hline Tumor grade ${ }^{b}$ & & & & 0.196 \\
\hline Low & 51 & 23 & 28 & \\
\hline High & 75 & 25 & 50 & \\
\hline
\end{tabular}

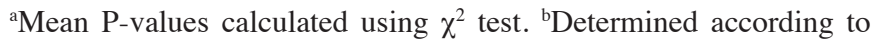
WHO 2004 classification (35). TRAF6, TNF receptor-associated factor 6 .

stage, tumor grade and metastasis (Table I). Notably, TRAF6 high expression was observed to be significantly associated with metastasis $(\mathrm{P}=0.001)$, indicating that high TRAF6 may promote the bladder cancer progression and metastasis. However, no significant associations between TRAF6 and other clinicopathological factors were observed.

Correlation between TRAF6 and recurrence rate. The correlation between TRAF6 and recurrence-free survival rate was first analyzed by Kaplan-Meier analysis (Table II). Only patients with tumor stage Ta-T1 $(n=97)$ were selected for analysis of rate of recurrence-free survival. In univariate analysis, recurrence-free survival rate was demonstrated to be significantly associated with tumor number and TRAF6 expression ( $\mathrm{P}=0.042$ and 0.016 , respectively; Fig. 2; Table II). Using the Cox-regression model in multivariate analysis, tumor number, tumor grade and TRAF6 expression were identified as independent predictive parameters of bladder cancer recurrence $(\mathrm{P}=0.008,0.029$ and 0.011 , respectively; Table II).

Effects of TRAF6 expression on patient outcome. The effects of TRAF6 expression on prognosis was subsequently evaluated by analyzing the correlation between TRAF6 expression and 5-year overall survival rates using Kaplan-Meier analysis (Table III). High TRAF6 expression was demonstrated to be significantly associated with poorer prognosis in patients with urothelial bladder cancer compared with low TRAF6 expression (univariate analysis, $\mathrm{P}=0.016,61.0$ vs. $47.8 \%$; Fig. $3 \mathrm{~A}$ ). Apart from TRAF6 expression, tumor number, metastasis status, tumor stage and tumor grade were also identified as prognostic factors in univariate analysis $(\mathrm{P}=0.003, \mathrm{P}<0.001$, $\mathrm{P}=0.001$ and $\mathrm{P}=0.023$, respectively; Fig. 3B-E).

Metastasis status was excluded from multivariate analysis, because it was indicated to be associated with TRAF6 expression using the $\chi^{2}$ test in univariate analysis (Table I). In multivariate analysis, TRAF6 expression was identified as an independent prognostic factor in urothelial bladder cancer (hazard ratio, 2.04; 95\% confidence interval, 0.92-4.49; $\mathrm{P}=0.037$; Table III). Furthermore, additional three factors, including tumor number, stage and grade, were also identified 
Table II. Univariate and multivariate analyses of association between clinicopathological factors and progression-free survival in patients with non-muscle-invasive bladder cancer.

\begin{tabular}{|c|c|c|c|c|c|}
\hline \multirow[b]{2}{*}{ Parameters } & \multicolumn{2}{|c|}{ Univariate analysis } & \multicolumn{3}{|c|}{ Multivariate analysis } \\
\hline & 5 -year recurrence-free rate, $\%$ & P-value ${ }^{a}$ & HR & $95 \% \mathrm{CI}$ & P-value ${ }^{b}$ \\
\hline Sex & & 0.926 & & & 0.498 \\
\hline Male & 26.4 & & 1 & & \\
\hline Female & 26.6 & & 1.24 & $0.67-2.30$ & \\
\hline Age & & 0.313 & & & 0.168 \\
\hline$<60$ & 40.8 & & 1 & & \\
\hline$\geq 60$ & 20.6 & & 1.51 & $0.84-2.69$ & \\
\hline Tumor diameter, $\mathrm{cm}$ & & 0.359 & & & 0.114 \\
\hline$\leq 3$ & 28.8 & & 1 & & \\
\hline$>3$ & 25.5 & & 1.55 & $0.90-2.66$ & \\
\hline Tumor number & & 0.042 & & & 0.008 \\
\hline Single & 34.9 & & 1 & & \\
\hline Multiple & 14.6 & & 2.12 & $1.22-3.68$ & \\
\hline Tumor grade & & 0.080 & & & 0.029 \\
\hline Low grade & 28.6 & & 1 & & \\
\hline High grade & 13.2 & & 2.15 & $1.08-4.29$ & \\
\hline TRAF6 expression & & 0.016 & & & 0.011 \\
\hline Low & 33.9 & & 1 & & \\
\hline High & 18.7 & & 2.04 & $1.18-3.53$ & \\
\hline
\end{tabular}

${ }^{a}$ Means calculated by Kaplan-Meier analysis and the log-rank test. ${ }^{b}$ Means calculated using the Cox-regression model. CI, confidence interval; HR, hazard ratio; TRAF6, TNF receptor-associated factor 6.

A

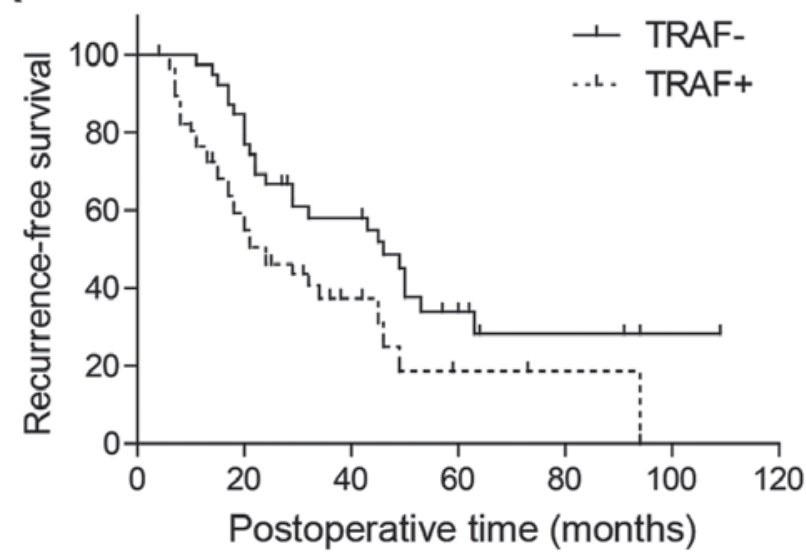

B

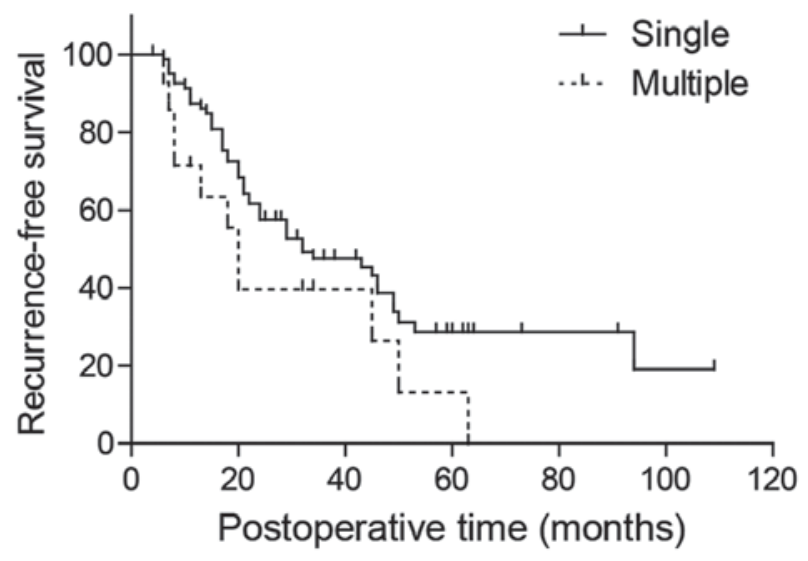

Figure 2. Kaplan-Meier survival curves of patients with urothelial bladder cancer displaying: (A) Correlation between TRAF6 expression and recurrence-free survival rate; (B) correlation between tumor number and recurrence-free survival rate as analyzed by univariate analysis. Vertical lines, censored cases.

to be independent prognostic factors $(\mathrm{P}=0.022,0.013$ and 0.030 , respectively; Table III).

\section{Discussion}

Bladder cancer was the most common malignancy of the urinary tract worldwide in 2014 and is also prominent for its high recurrence rate in all treated patients (26). Investigating potential biomarkers may bring about new breakthroughs in strategies on reducing recurrence and survival rate, similar to the discovery of trastuzumab following studies on the human epidermal growth factor receptor-2 (HER2) studies.

Recently, important progress has been made in the biomarker field particularly in new detection techniques in genomics and proteomics $(27,28)$. In bladder cancer, a number of accomplishments were achieved, including the 
Table III. Associations between clinicopathological factors and prognosis in urothelial bladder cancer.

\begin{tabular}{|c|c|c|c|c|c|}
\hline \multirow[b]{2}{*}{ Parameters } & \multicolumn{2}{|c|}{ Univariate analysis } & \multicolumn{3}{|c|}{ Multivariate analysis } \\
\hline & 5 -year survival rate, $\%$ & P-value ${ }^{a}$ & HR & $95 \% \mathrm{CI}$ & P-value ${ }^{b}$ \\
\hline Sex & & 0.669 & & & 0.336 \\
\hline Male & 53.9 & & 1 & & \\
\hline Female & 48.6 & & 1.47 & $0.67-3.24$ & \\
\hline Age & & 0.804 & & & 0.629 \\
\hline$<60$ & 57.4 & & 1 & & \\
\hline$\geq 60$ & 41.7 & & 0.83 & $0.38-1.78$ & \\
\hline Tumor diameter, $\mathrm{cm}$ & & 0.436 & & & 0.188 \\
\hline$\leq 3$ & 54.7 & & 1 & & \\
\hline$>3$ & 54.4 & & 1.61 & $0.79-3.25$ & \\
\hline Tumor number & & 0.003 & & & 0.022 \\
\hline Single & 61.4 & & 1 & & \\
\hline Multiple & 17.2 & & 2.47 & $0.96-6.35$ & \\
\hline Metastasis status & & $<0.001$ & & & \\
\hline Negative & 55.8 & & - & & \\
\hline Positive & 0.0 & & - & - & - \\
\hline Tumor stage & & 0.001 & & & 0.013 \\
\hline Ta-T1 & 58.0 & & 1 & & \\
\hline $\mathrm{T} 2-\mathrm{T} 4$ & 23.6 & & 4.05 & $1.35-12.17$ & \\
\hline Tumor grade & & 0.023 & & & 0.030 \\
\hline Low grade & 63.6 & & 1 & & \\
\hline High grade & 43.7 & & 2.33 & $1.09-4.99$ & \\
\hline TRAF6 expression & & 0.016 & & & 0.037 \\
\hline Low & 61.0 & & 1 & & \\
\hline High & 47.8 & & 2.04 & $0.92-4.49$ & \\
\hline
\end{tabular}

${ }^{a}$ Means calculated using Kaplan-Meier analysis and the log-rank test. ${ }^{b}$ Means calculated using the Cox-regression model. CI, confidence interval; HR, hazard ratio; TRAF6, TNF receptor-associated factor 6.

identification of the gene signatures, which associate with bladder cancer progression (29). However, basic science research is not sufficient for breakthroughs in bladder cancer treatment. Translational studies, including the study of potential biomarkers, also have an important role in advancing progress in treatment.

The identification of prognostic and predictive markers is of great importance to improve the management of patients and to decrease the rate of mortality. Promising biomarkers may lead to effective therapeutic drug targets and drugs.

TRAF6 has been demonstrated to mediate signal transduction from the TNF receptor superfamily and receptors, including cluster of differentiation 40, tumor necrosis factor superfamily member 11 and IL-1 (10). These receptors are involved in numerous signaling pathways and tumor processes. Furthermore, ectopic TRAF6 expression was observed in a number of types of cancer, including glioma, pancreas cancer $(9,16)$. However, the underlying molecular mechanisms in these types of cancer remain to be elucidated.

In bladder cancer, no previous study on TRAF6 function was reported to the best of our knowledge. However, in a large screen of effective genetic variants in microRNAs (miRNAs) in bladder cancer, TRAF6 was hypothesized as an effector molecule of miR-146a. miR-146a has an notable role in predicting the risk and recurrence of bladder cancer (30). This supported the results in the present study, which indicated that TRAF6 expression may be a prognostic factor in urothelial bladder cancer.

There are two main intracellular protein degradation pathways in eukaryotic cells: Ubiquitination and autophagy (31). Ubiquitination is an important post-translational modification that involves the multi-stage stepwise conjugation of proteins to ubiquitin monomers by the ubiquitinating enzymes E1, E2, and E3 (32). In this process, TRAF6 acts as an E3 ubiquitin ligase, which mediates the conjugation of lysine-63(K63) -linked polyubiquitin chains to targeted proteins, which is responsible for substrate specificity (10). The substrates of TRAF6-mediated ubiquitination include: IKBKG, IRAK1, AKT1 and AKT2 (33). In breast cancer, TRAF6 was demonstrated to ubiquitinate the proform of asparaginyl endopeptidase through K63-linked polyubiquitin therefore promoting invasion and metastasis in breast cancer (34). However, whether TRAF6 functions as an oncogenic factor in bladder cancer by facilitating the ubiquitination of a 

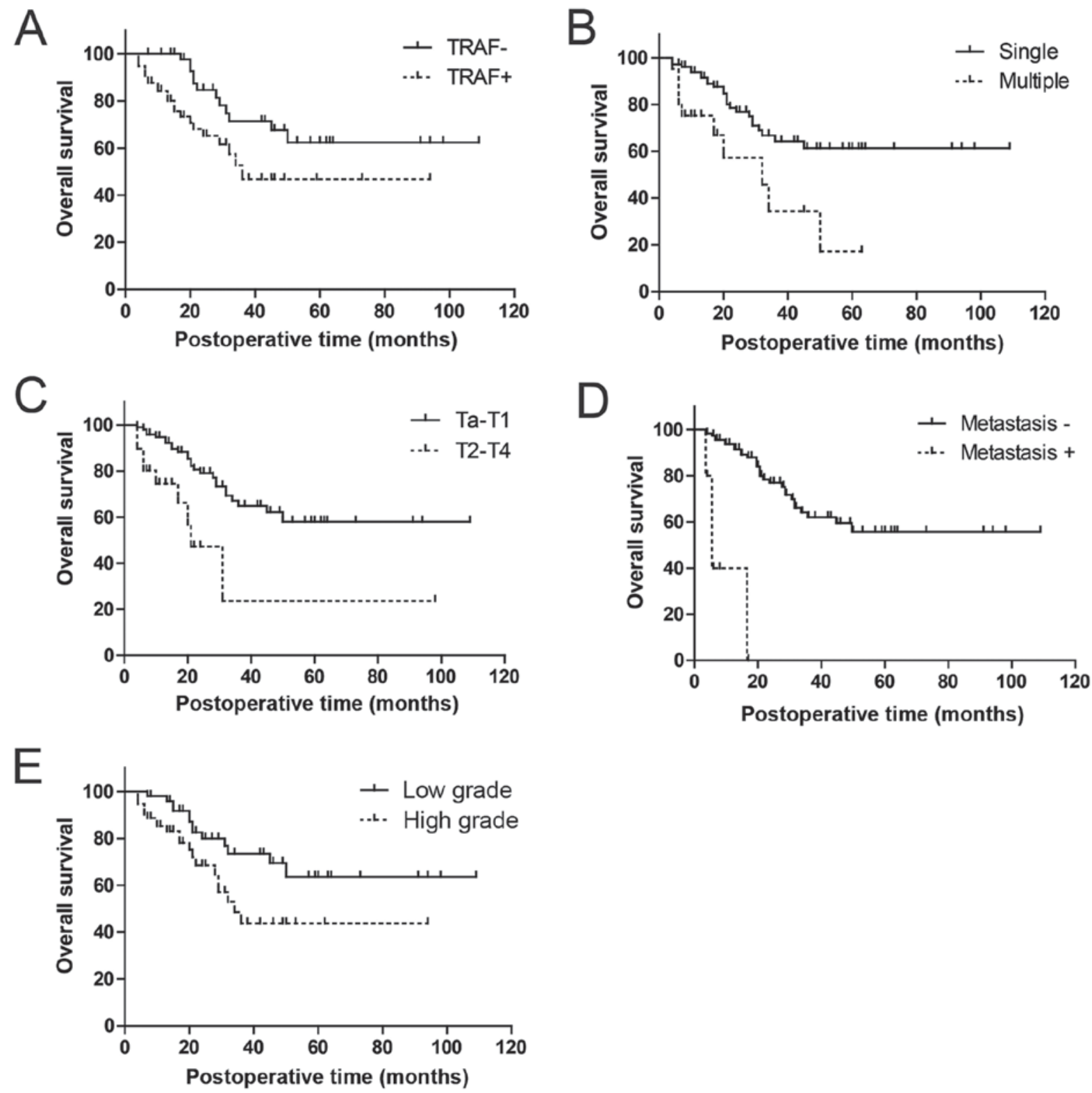

Figure 3. Kaplan-Meier survival curves of patients with urothelial bladder cancer presenting: (A) Correlation between TRAF6 expression and overall survival rate; (B) correlation between tumor number and overall survival rate; (C) correlation between tumor stage and overall survival rate; (D) correlation between metastasis and overall survival rate and $(\mathrm{E})$ correlation between tumor grade and overall survival rate.

downstream key molecule remain unknown. The authors of the present study.

The finding in the present study that TRAF6 overexpression is associated with the higher recurrence and poorer prognosis may trigger further interests in researching the underlying function of TRAF6 in bladder cancer and further investigation into potential chemotherapeutics for the treatment of bladder cancer.

In conclusion, TRAF6 expression was detected in 126 urothelial bladder cancer samples, and the clinical significance of TRAF6 expression was analyzed. It was demonstrated that TRAF6 expression was significantly associated with metastasis status, indicating that TRAF6 may promote the tumor invasion. In univariate and multivariate analysis, it was indicated that.

TRAF6 expression was an independent factor, which may predict bladder cancer recurrence and prognosis.

\section{Acknowledgements}

The present study was supported by Science and Technology Project of Yantai City (grant nos. 2015WS018 and 2016WS006), Youth Research Initiation Foundation of Yuhuangding Hospital (grant no. 201511) and Shandong Province Medical Science and Technology development project (grant no. 2016WS0706).

\section{References}

1. Torre LA, Bray F, Siegel RL, Ferlay J,Lortet-Tieulent J and Jemal A: Global cancer statistics, 2012. CA Cancer J Clin 65: 87-108, 2015.

2. Dy GW, Gore JL, Forouzanfar MH, Naghavi M and Fitzmaurice C: Global burden of urologic cancers, 1990-2013. Eur Urol 71: 437-446, 2017.

3. La Vecchia C, Bosetti C, Lucchini F, Bertuccio P, Negri E, Boyle P and Levi F: Cancer mortality in Europe, 2000-2004, and an overview of trends since 1975. Ann Oncol 21: 1323-1360, 2010. 
4. Jiang X, Du L, Wang L, Li J, Liu Y, Zheng G, Qu A, Zhang X, Pan $\mathrm{H}$, Yang Y and Wang C: Serum microRNA expression signatures identified from genome-wide microRNA profiling serve as novel noninvasive biomarkers for diagnosis and recurrence of bladder cancer. Int J Cancer 136: 854-862, 2015.

5. Babjuk M, Oosterlinck W, Sylvester R, Kaasinen E, Böhle A, Palou-Redorta J and Rouprêt M; European Association of Urology (EAU): EAU guidelines on non-muscle-invasive urothelial carcinoma of the bladder, the 2011 update. Eur Urol 59: 997-1008, 2011.

6. Wu XR: Urothelial tumorigenesis: A tale of divergent pathways. Nat Rev Cancer 5: 713-725, 2005.

7. Black PC, Brown GA and Dinney CP: Molecular markers of urothelial cancer and their use in the monitoring of superficial urothelial cancer. J Clin Oncol 24: 5528-5535, 2006.

8. Cheng L, Zhang S, MacLennan GT, Williamson SR, Lopez-Beltran A and Montironi R: Bladder cancer: Translating molecular genetic insights into clinical practice. Hum Pathol 42 : 455-481, 2011

9. Chiu HW, Lin SW, Lin LC, Hsu YH, Lin YF, Ho SY, Wu YH and Wang YJ: Synergistic antitumor effects of radiation and proteasome inhibitor treatment in pancreatic cancer through the induction of autophagy and the downregulation of TRAF6. Cancer Lett 365: 229-239, 2015.

10. Deng L, Wang C, Spencer E, Yang L, Braun A, You J, Slaughter C, Pickart $\mathrm{C}$ and Chen ZJ: Activation of the IkappaB kinase complex by TRAF6 requires a dimeric ubiquitin-conjugating enzyme complex and a unique polyubiquitin chain. Cell 103: 351-361, 2000

11. Chen H, Li M, Campbell RA, Burkhardt K, Zhu D, Li SG, Lee HJ, Wang C, Zeng Z, Gordon MS, et al: Interference with nuclear factor kappa B and c-Jun NH2-terminal kinase signaling by TRAF6C small interfering RNA inhibits myeloma cell proliferation and enhances apoptosis. Oncogene 25: 6520-6527, 2006.

12. Wang KZ, Galson DL and Auron PE: TRAF6 is autoinhibited by an intramolecular interaction which is counteracted by trans-ubiquitination. J Cell Biochem 110: 763-771, 2010.

13. Lamothe B, Besse A, Campos AD, Webster WK, Wu H and Darnay BG: Site-specific Lys-63-linked tumor necrosis factor receptor-associated factor 6 auto-ubiquitination is a critical determinant of I kappa B kinase activation. J Biol Chem 282: 4102-4112, 2007

14. Choi YB and Harhaj EW: HTLV-1 tax stabilizes MCL-1 via TRAF6-dependent K63-linked polyubiquitination to promote cell survival and transformation. PLoS Pathog 10: e1004458, 2014.

15. Chaudhry SI, Hooper S, Nye E, Williamson P, Harrington K and Sahai E: Autocrine IL-1 $\beta$-TRAF6 signalling promotes squamous cell carcinoma invasion through paracrine $\mathrm{TNF} \alpha$ signalling to carcinoma-associated fibroblasts. Oncogene 32: 747-758, 2013.

16. Liu J, Xu J, Li H, Sun C, Yu L, Li Y, Shi C, Zhou X, Bian X, Ping Y, et al: miR-146b-5p functions as a tumor suppressor by targeting TRAF6 and predicts the prognosis of human gliomas. Oncotarget 6: 29129-29142, 2015.

17. Rong Y, Wang D, Wu W, Jin D, Kuang T, Ni X, Zhang L and Lou W: TRAF6 is over-expressed in pancreatic cancer and promotes the tumorigenicity of pancreatic cancer cells. Med Oncol 31: 260, 2014

18. Liu H, Zhang T, Ye J, Li H, Huang J, Li X, Wu B, Huang X and Hou J: TNF receptor-associated factor 6 in advanced non-small cell lung cancer: Clinical and prognostic implications. J Cancer Res Clin Oncol 138: 1853-1863, 2012.
19. Sanli O, Dobruch J, Knowles MA, Burger M, Alemozaffar M, Nielsen ME and Lotan Y: Bladder cancer. Nat Rev Dis Primers 3: $17022,2017$.

20. Liu H, Xu Y, Zhang Q, Yang H, Shi W, Liu Z, Li K, Gong Z, Ning S, Li S and Chen Y: Prognostic significance of TBL1XR1 in predicting liver metastasis for early stage colorectal cancer. Surg Oncol 26: 13-20, 2017.

21. Xu YF, Yang XQ, Lu XF, Guo S, Liu Y, Iqbal M, Ning SL, Yang H, Suo N and Chen YX: Fibroblast growth factor receptor 4 promotes progression and correlates to poor prognosis in cholangiocarcinoma. Biochem Biophys Res Commun 446: 54-60, 2014.

22. Xu Y, Yang X, Li Z, Li S, Guo S, Ismail S, Liu H, Huang Z, Zhang Z, Chen Y and Sun Q: Sprouty2 correlates with favorable prognosis of gastric adenocarcinoma via suppressing FGFR2-induced ERK phosphorylation and cancer progression. Oncotarget 8: 4888-4900, 2017.

23. Hemdan T, Lindén M, Lind SB, Namuduri AV, Sjöstedt E, de Ståhl TD, Asplund A, Malmström PU and Segersten U: The prognostic value and therapeutic target role of stathmin-1 in urinary bladder cancer. Br J Cancer 111: 1180-1187, 2014

24. Yang XQ, Xu YF, Guo S, Liu Y, Ning SL, Lu XF, Yang H and Chen YX: Clinical significance of nerve growth factor and tropomyosin-receptor-kinase signaling pathway in intrahepatic cholangiocarcinoma. World J Gastroenterol 20: 4076-4084, 2014

25. Liu H, Xu Y, Zhang Q, Li K, Wang D, Li S, Ning S, Yang H, Shi W, Liu Z and Chen Y: Correlations between TBL1XR1 and recurrence of colorectal cancer. Sci Rep 7: 44275, 2017.

26. Solomon JP and Hansel DE: Prognostic factors in urothelial carcinoma of the bladder: Histologic and molecular correlates. Adv Anat Pathol 22: 102-112, 2015.

27. Gogalic S, Sauer U, Doppler S and Preininger C: Bladder cancer biomarker array to detect aberrant levels of proteins in urine. Analyst 140: 724-735, 2015.

28. Chan EC, Pasikanti KK, Hong Y, Ho PC, Mahendran R, Raman Nee Mani L, Chiong E and Esuvaranathan K: Metabonomic profiling of bladder cancer. J Proteome Res 14: 587-602, 2015.

29. Mitra AP, Bartsch CC and Cote RJ: Strategies for molecular expression profiling in bladder cancer. Cancer Metastasis Rev 28: 317-326, 2009.

30. Wang M, Chu H, Li P, Yuan L, Fu G, Ma L, Shi D, Zhong D, Tong N, Qin C, et al: Genetic variants in miRNAs predict bladder cancer risk and recurrence. Cancer Res 72: 6173-6182, 2012.

31. Shaid S, Brandts $\mathrm{CH}$, Serve $\mathrm{H}$ and Dikic I: Ubiquitination and selective autophagy. Cell Death Differ 20: 21-30, 2013.

32. Pickart CM: Ubiquitin enters the new millennium. Mol Cell 8 : 499-504, 2001

33. Yang WL, Wang J, Chan CH, Lee SW, Campos AD, Lamothe B, Hur L, Grabiner BC, Lin X, Darnay BG and Lin HK: The E3 ligase TRAF6 regulates Akt ubiquitination and activation. Science 325: 1134-1138, 2009.

34. Lin Y, Qiu Y, Xu C, Liu Q, Peng B, Kaufmann GF, Chen X, Lan B, Wei C, Lu D, et al: Functional role of asparaginyl endopeptidase ubiquitination by TRAF6 in tumor invasion and metastasis. J Natl Cancer Inst 106: dju012, 2014. 